\title{
Neuropsychiatric Manifestations of Systemic Lupus Erythematosus - A Case Report
}

\section{Viraj Shah $^{1 *}$ | Jonathan T. Grossman ${ }^{2}$ | Priya Patel ${ }^{1}$ | Daniel Gutman ${ }^{1}$}

*Correspondence: Viraj Shah

Address: ${ }^{1}$ The Florida Atlantic University Charles E. Schmidt College of Medicine Internal Medicine Residency Program; Boca Raton, Florida; ${ }^{2}$ The Florida Atlantic University Charles E. Schmidt College of Medicine Neurology Residency Program; Boca Raton, Florida

e-mail $\bowtie$ : vshah@health.fau.edu

Received: 16 August 2021; Accepted: 23 August 2021

Copyright: (C) 2021 Shah V. This is an open-access article distributed under the terms of the Creative Commons Attribution License, which permits unrestricted use, distribution, and reproduction in any medium, provided that the original work is properly cited.

\section{ABSTRACT}

Systemic lupus erythematosus (SLE) is known for marked heterogeneity of clinical manifestations and pathogenesis with the potential of affecting virtually every organ system. Neuropsychiatric manifestations of systemic lupus erythematosus are difficult to recognize and treat as they remain one of the least understood complications of this disease. Our case describes a 42-year-old female who presented to the emergency department with proximal weakness of the left lower extremity, magnetic resonance imaging (MRI) of the brain revealed a small infarct in the medial left temporal lobe. Workup was remarkable for elevated inflammatory markers. The patient was discharged on appropriate medical therapy however returned to the emergency department one month later after a witnessed focal seizure. Neuroimaging was consistent with cerebral vasculitis and autoimmune workup revealed a diagnosis of SLE. Our case highlights the importance of recognizing that neuropsychiatric manifestations of SLE often occur in absence of other systemic manifestations and can be the initial presentation of SLE. Maintaining a high clinical suspicion for Neuropsychiatric Systemic Lupus Erythematosus (NPSLE) could afford the ability to halt disease progression in the earliest of phases and thus improve quality of life through early administration of appropriate pharmacotherapy.

Keywords: Lupus, Systemic Lupus Erythematosus, SLE, Neuropsychiatric, Cerebral Vasculitis, Seizure, American College of Rheumatology

\section{Introduction}

Systemic lupus erythematosus (SLE) is a chronic autoimmune disease characterized by autoantibody production and immune complex formation. There is a marked heterogeneity of clinical manifestations and its pathogenesis and epidemiological burden spans the globe; it has affected virtually every demographic and has no boundary with regard to race, ethnicity, age, or gender (Stojan and Petri, 2018). Renal and central nervous system (CNS) involvement accompanies severe disease and accounts for a large proportion of morbidity and mortality (Cervera et al., 2003). Neuropsychiatric manifestations in SLE are estimated to occur in approximately 14 to $80 \%$ of patients (Kivity et al., 2015). Most 
importantly, neuropsychiatric symptoms can be the initial presenting symptom and often in absence of other accompanying systemic signs or symptoms. We present the case of a 42-year-old female who presented with neuropsychiatric symptoms in the context of acute ischemic stroke and focal seizure as initial manifestations of SLE.

\section{Case Description}

A 42-year-old African American female without significant past medical history presented to our emergency department with generalized weakness in the setting of recurrent syncope for three weeks. She had also been increasingly forgetful with fluctuant mood. On initial physical examination, she was noted to have proximal weakness of the left lower extremity with $3 / 5$ strength upon hip flexion. Her sensory exam was unremarkable. Of note, there was no evidence of oral lesions or skin rash. Vital signs including orthostatics were unremarkable. Initial labs were as follows: hemoglobin $9.9 \mathrm{~g} / \mathrm{dl}$ [normal range 12-15.5], WBC count 3300/microliter [N 4.5-11], creatinine $0.7 \mathrm{mg} / \mathrm{dl}$ [N 0.59-1.04], ESR 119 $\mathrm{mm} / \mathrm{hr}$ [N 0-29], CRP $0.32 \mathrm{mg} / \mathrm{dl}$ [N < 10]. Urine analysis did not show any proteinuria. Non-enhanced CT scan of head was unremarkable however MRI brain revealed a small infarct in the medial left temporal lobe in the setting of subcortical white matter changes secondary to chronic small vessel disease. A hypercoaguable panel including Factor V Leiden, Protein $\mathrm{C}$ and $\mathrm{S}$, antithrombin antibody, lupus anticoagulant, and antiphospholipid antibody was unremarkable. She was initiated on dual antiplatelet therapy via aspirin and clopidogrel as well as atorvastatin and subsequently discharged. Unfortunately, she returned 1 month later following a witnessed focal seizure of the left upper extremity. Remarkable labs were as follows: hemoglobin of $10.1 \mathrm{~g} / \mathrm{dl}$, WBC count 3400/microliter, creatinine $0.6 \mathrm{mg} / \mathrm{dl}$. Autoimmune workup was remarkable for persistently elevated ESR of $111 \mathrm{~mm} / \mathrm{hr}$, complement component 3 (C3) $40 \mathrm{mg} / \mathrm{dl}$ [N 88-201], complement component 4 (C4) $8.4 \mathrm{mg} / \mathrm{dl}$ [N 14-45], and ANA of 1:320 (low titers $=1: 40$ to $1: 60$ ) with a speckled pattern of distribution. Anti-double-stranded DNA (dsDNA) was positive with a titer of 1:640 ( $<30 \mathrm{IU} / \mathrm{mL})$. Other autoantibodies including anti-smith, anti-ribosomal P, anti-U1RNP, anti-SS-A, and anti-SS-B were negative (Mummert et al., 2018). MRI of the brain with contrast revealed multiple scattered foci of restricted diffusion in bilateral posterior MCA territories with subcortical white matter changes that appeared consistent with cerebral vasculitis (Fig. 1).

A diagnosis of probable SLE was made per the 2019 European League Against Rheumatism (EULAR)/American Academy of Rheumatology (ACR) classification criteria. She was started on Levetiracetam 500mg twice daily, Hydroxychloroquine 200mg twice daily, and prednisone 60mg daily (Fanouriakis et al., 2019). She had no further seizures during her stay. She was discharged with recommendation to establish care with rheumatology on an outpatient basis. 


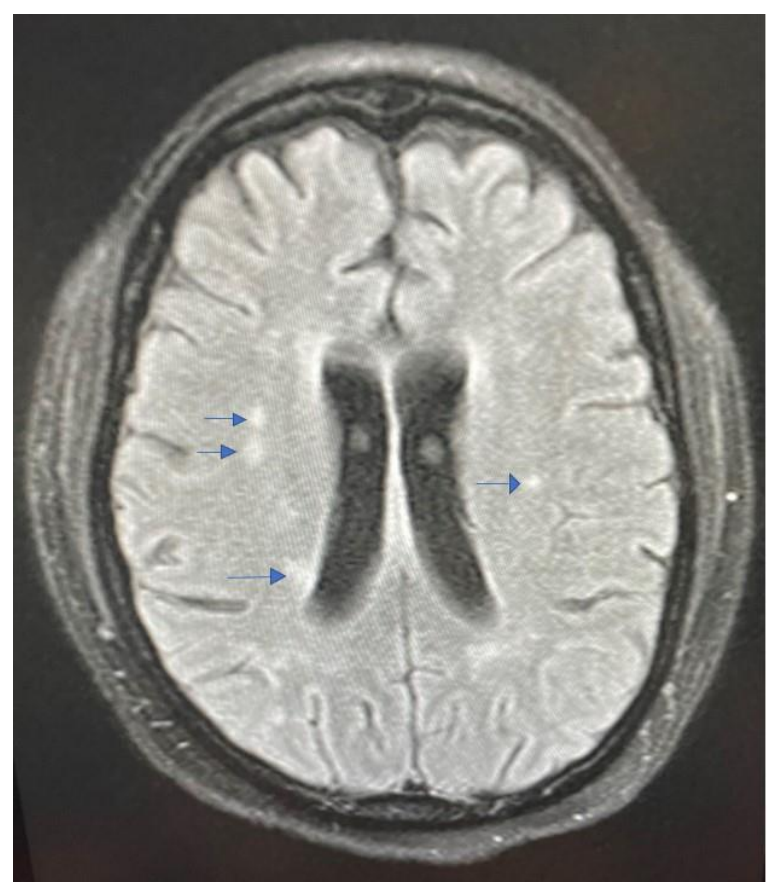

Figure 1: MRI brain axial T2-weighted image showing multiple hyperintense foci.

\section{Discussion}

At least five million people worldwide have SLE and it is estimated that up to $75 \%$ may develop neuropsychiatric manifestations; this is also known as neuropsychiatric systemic lupus erythematosus or NPSLE. The evolution of NPSLE is thought to be secondary to many factors culminating in the generation of autoantibodies with immune complex deposition and a cascade of inflammatory cytokines leading to neuronal injury. It may be an exceedingly prevalent, often overlooked manifestation of SLE as it can present in absence of traditional serologic markers. While immunosuppressants can eventually confer improvement in neuropsychiatric symptoms, many patients could benefit from adjunctive treatment that specifically addresses psychosis, cognitive dysfunction, and disordered mood. It is crucial to recognize NPSLE as it has been associated with increased morbidity and mortality (Hanly et al., 2005).

According to the 1999 American College of Rheumatology classification, neurological manifestations of SLE include cerebrovascular disease, seizures, aseptic meningitis, headache, cognitive dysfunction, mood disorder, psychosis, movement disorders, Guillain-Barre syndrome, and autonomic neuropathy (Jafri et al., 2017). Neuropsychiatric manifestations of systemic lupus erythematosus are difficult to recognize and treat as they remain one of the least understood complications of this disease. In approximately $28-40 \%$ of adults, neuropsychiatric symptoms develop just before subsequent diagnosis of SLE (Hanly et al., 2007). However, the diagnosis is often missed due to absence of serologic markers or evidence of disease activity outside of the nervous system. Studies in adults using the case definitions per ACR have detected the presence of anywhere from 14 to 17 out of the 19 signs/symptoms as follows: 
headache (39\% to $61 \%$ ), seizures ( $8 \%$ to $18 \%$ ), cerebrovascular disease ( $2 \%$ to $8 \%$ ), psychosis $(3 \%$ to 5\%), cranial neuropathy (1.5\% to 2.1\%), and movement disorders (1\%) (Carrión-Barberà I et al., 2021). The pathogenesis of NPSLE appears to be multifactorial. It includes blood-brain barrier dysfunction, microangiopathy, atherosclerosis, as well as direct neuronal cell damage mediated by autoantibodies, complement components, and cytokines. Numerous autoantibodies have been implicated in the evolution of NPSLE and targets include the following: phospholipids, ribosomal-P peptides (Rib-P), endothelial cells, glutamate receptors, microtubule-associated protein 2, and MMP-9 (Brey, 2007). Current therapeutic strategies in NPSLE include the use of immunosuppressive therapies when the underlying pathogenesis is considered primarily inflammatory or in cases where there is evidence of generalized SLE activity (Fanouriakis et al., 2007).

Our patient presented with stroke as the presumed initial manifestation of SLE. We suspect that her second admission for focal seizure could possibly have been prevented had she been started on immunosuppressants after appropriate autoimmune work-up during her first admission. With regard to her fluctuant mood described by family prior to arrival, we did not observe this during either stay and therefore recommended that she establish care with outpatient psychological/psychiatric services upon discharge.

\section{Conclusion}

It is crucial to recognize that neuropsychiatric manifestations of SLE often occur in absence of other systemic manifestations and can be the initial presentation of SLE, especially because NPSLE has been associated with a worse prognosis than traditional SLE. A multidisciplinary approach can afford early recognition when it may be more feasible to halt disease progression.

\section{References}

Brey RL. Neuropsychiatric lupus: clinical and imaging aspects. Bull NYU Hosp Jt Dis 2007; 65: 194-199.

Carrión-Barberà I, Salman-Monte TC, Vílchez-Oya F, Monfort J. Neuropsychiatric involvement in systemic lupus erythematosus: A review. Autoimmun Rev 2021; 20: 102780.

Cervera R, Khamashta MA, Font J, Sebastiani GD, Gil A, Lavilla P, Mejía JC, Aydintug AO, Chwalinska-Sadowska H, de Ramón E, Fernández-Nebro A. Morbidity and mortality in systemic lupus erythematosus during a 10-year period: a comparison of early and late manifestations in a cohort of 1,000 patients. Medicine (Baltimore) 2003; 82: 299-308.

Fanouriakis A, Kostopoulou M, Alunno A, Aringer M, Bajema I, Boletis JN, Cervera R, Doria A, Gordon C, Govoni M, Houssiau F. 2019 update of the EULAR recommendations for the management of systemic lupus erythematosus. Ann Rheum Dis 2019; 78: 736-745.

Govoni M, Bortoluzzi A, Padovan M, Silvagni E, Borrelli M, Donelli F, Ceruti S, Trotta F. The diagnosis and clinical management of the neuropsychiatric manifestations of lupus. J Autoimmun 2016; 74: 41-72. 
Hanly JG, Urowitz MB, Sanchez-Guerrero J, Bae SC, Gordon C, Wallace DJ, Isenberg D, Alarcon GS, Clarke A, Bernatsky S, Merrill JT. Neuropsychiatric events at the time of diagnosis of systemic lupus erythematosus: an international inception cohort study. Arthritis Rheum 2007; 56: 265-273.

Jafri K, Patterson SL, Lanata C. Central Nervous System Manifestations of Systemic Lupus Erythematosus. Rheum Dis Clin North Am 2017; 43: 531-545.

Kivity S, Agmon-Levin N, Zandman-Goddard G, Chapman J, Shoenfeld Y. Neuropsychiatric lupus: a mosaic of clinical presentations. BMC Med 2015; 13: 43.

Mummert E, Fritzler MJ, Sjöwall C, Bentow C, Mahler M. The clinical utility of anti-double-stranded DNA antibodies and the challenges of their determination. J Immunol Methods 2018; 459: 11-19.

Stojan G and Petri M. Epidemiology of systemic lupus erythematosus: an update. Curr Opin Rheumatol 2018; 30: 144-150. 